\section{ORIGINAL RESEARCH}

\section{R. Della Nave \\ S. Foresti \\ A. Pratesi \\ A. Ginestroni \\ M. Inzitari \\ E. Salvadori \\ M. Giannelli \\ S. Diciotti \\ D. Inzitari}

M. Mascalchi

\title{
Whole-Brain Histogram and Voxel-Based Analyses of Diffusion Tensor Imaging in Patients with Leukoaraiosis: Correlation with Motor and Cognitive Impairment
}

\begin{abstract}
BACKGROUND AND PURPOSE: Cerebral white matter changes, termed leukoaraiosis (LA), appearing as areas of increased signal intensity in T2-weighted MR images, are common in elderly subjects, but the possible correlation of LA with cognitive or motor deficit has not been established. We hypothesized that histogram and voxel-based analyses of whole-brain mean diffusivity (MD) and fractional anisotropy (FA) maps calculated from diffusion tensor imaging (DTI) could be more sensitive tools than visual scales to investigate the clinical correlates of LA.
\end{abstract}

\begin{abstract}
MATERIALS AND METHODS: Thirty-six patients of the Leukoaraiosis and Disability Study were evaluated with fluid-attenuated inversion recovery for LA extension, T1-weighted images for volume, and DTI for MD and FA. The extent of LA was rated visually. The normalized total, gray, and white matter brain volumes were computed, as well as the 25th percentile, 50th percentile, kurtosis, and skewness of the MD and FA maps of the whole brain. Finally, voxel-based analysis on the maps of gray and white matter volume, MD, and FA was performed with SPM2 software. Correlation analyses between visual or computerized data and motor or neuropsychologic scale scores were performed using the Spearman rank test and the SPM2 software.
\end{abstract}

\begin{abstract}
RESULTS: The visual score correlated with some MD and FA histogram metrics $(P<.01)$. However, only the 25th and 50th percentiles, kurtosis, and skewness of the MD and FA histograms correlated with motor or neuropsychologic deficits. Voxel-based analysis revealed a correlation $(P<.05$ corrected for multiple comparisons) between a large cluster of increased MD in the corpus callosum and pericallosal white matter and motor deficit.
\end{abstract}

CoNCLUSIONS: These results are consistent with the hypothesis that histogram and voxel-based analyses of the whole-brain MD and FA maps are more sensitive tools than the visual evaluation for clinical correlation in patients with LA.

c erebral white matter changes, appearing as hypoattenuated areas in CT scans and as hyperintense areas in T2weighted MR images, are common in elderly subjects and have been termed leukoaraiosis (LA). The correlation of LA with arterial hypertension and history of stroke and some neuropathologic studies suggest a small vessel alteration as the underlying cause of LA. ${ }^{-3}$ However, LA also correlates with aging and is a nonspecific finding, because it reflects different histopathologic changes, such as demyelination, loss or increase of glial cells, vacuolization (spongiosis), apoptosis of oligodendrocytes, and wallerian degeneration. ${ }^{3,4}$ The possible correlation of LA with cognitive decline or motor deficit has been investigated using visual or computerized methods to assess the LA extent. ${ }^{5-16}$ Diffusion-weighted imaging (DWI) and diffusion tensor imaging (DTI) are MR techniques sensitive to changes in the diffusion properties of water protons in terms of apparent diffusion coefficient (ADC), mean diffusivity

\section{Received October 16, 2006; accepted after revision November 16.}

From the Radiodiagnostic Section, Department of Clinical Physiopathology (R.D.N., S.F., A.P., A.G., M.M.), Clinica Neurologica III, Department of Neurology and Psychiatry (M.I., E.S., D.I.), and Department of Electronics and Telecommunications (S.D.), University of Florence, Florence, Italy; Medical Physics (M.G.), Azienda Ospedaliera Universitaria Pisana, Pisa, Italy.

Address correspondence to Mario Mascalchi, Sezione di Radiodiagnostica, Dipartimento di Fisiopatologia Clinica, University of Florence, Florence, Italy; e-mail: m.mascalchi@dfc. unifi.it

Indicates article with supplemental on-line table.

DOI 10.3174/ajnr.A0555
(MD), or fractional anisotropy (FA). Previous studies using region of interest measurements have demonstrated that MD and FA in patients with LA are altered not only in the areas exhibiting signal intensity changes in $\mathrm{T} 2$-weighted images but also in the normal-appearing white matter. ${ }^{17,18}$ Using wholebrain histogram analysis of ADC, it is possible to obtain a quantitative global assessment of the tissue changes associated with LA. ${ }^{19,20}$ Recently, voxel-based analysis has also emerged as a powerful tool to investigate regional changes of the volume, $\mathrm{ADC}, \mathrm{MD}$, or FA of the whole brain in a number of diseases. ${ }^{21-24}$ We hypothesized that histogram and voxelbased analyses of whole-brain MD and FA maps calculated from DTI could be more sensitive tools than visual scales to investigate the clinical correlates of LA. Accordingly, we used histogram and voxel-based analyses to measure the global and regional changes in 36 patients with LA belonging to the Leukoaraiosis and Disability (LADIS) Study who were examined with DTI and correlated the findings obtained with these methods, with 1 visual scale, and with the results of quantitative motor and neuropsychologic evaluations.

\section{Materials and Methods}

\section{The LADIS Study}

The LADIS Study is a longitudinal multicentric European study, which aims: 1) to establish whether LA and its progression over time play a role as an independent determinant of the transition from functional autonomy to disability in elderly subjects; 2 ) to confirm 


\begin{tabular}{|c|c|c|c|c|}
\hline Variable & Mean & SD & Range & $\begin{array}{l}\text { Normal } \\
\text { Values }\end{array}$ \\
\hline$\overline{\text { SPPB }}$ & 8 & 2.5 & $4-12$ & $12^{*}$ \\
\hline Usual gait velocity, $\mathrm{m} / \mathrm{s}$ & 3 & 1 & $1-4$ & $1.2 \pm 0.2 \dagger$ \\
\hline Single-leg stance time, s & 15 & 10.8 & $1-41$ & $17.2 \pm 4.1 \dagger$ \\
\hline MMSE & 26 & 3.4 & $17-30$ & $\geq 27$ \\
\hline Stroop Test & 57 & 25.3 & 26-158 & NA \\
\hline Trail Making Test, s & 90 & 49 & $33-225$ & NA \\
\hline Maze Task, s & 9 & 7 & $2-33$ & NA \\
\hline Digit Test & 13 & 4.3 & $2-22$ & NA \\
\hline Verbal Fluency Test & 15 & 4.8 & $6-25$ & NA \\
\hline
\end{tabular}

Note:-SPPB indicates Short Physical Performance Battery; MMSE, Mini Mental State Examination; LA, leukoaraiosis; NA, not available.

* From reference 26.

† From reference 8.

whether LA and its progression independently predict death, cardiovascular events, and dementia; 3 ) to examine whether progression of LA parallels the deterioration of specific motor and cognitive performances; and 4) to evaluate whether LA has an impact on quality of life. ${ }^{25}$ The enrollment criteria of the LADIS Study are detailed elsewhere $^{25}$ and include the following: 1) age between 64 and 84 years; 2) changes of the cerebral white matter on MR imaging of any degree; and 3) no or mild disability. The study design establishes a core MR imaging protocol including T1 and T2-weighted fluid-attenuated inversion recovery (FLAIR) images that is shared by the 11 participating centers, whereas DWI, DTI, or magnetization transfer techniques are used by selected centers to further characterize tissue changes.

\section{Subjects}

The study was approved by our institutional review board. Thirty-six consecutive patients ( 21 women and 15 men; mean age, $77 \pm 4.5$ years; age range, 69-84 years) belonging to the LADIS Study were examined. None of them had evidence of corticosubcortical infarcts. All 36 of the patients were evaluated according to the LADIS protocol, which includes a functional and clinical assessment. ${ }^{25}$

In particular, 1 of the authors with 6 years of clinical experience in geriatric neurology (M.I.) evaluated all of the patients for motor deficit with a modified Short Physical Performance Battery (SPPB), ${ }^{26}$ in which the original " 8 -foot-walk" was substituted by a "4-m-walk" test, and with 2 simple measures of gait and balance (gait velocity and single-leg stance time). ${ }^{4}$ Increasing deficits correspond with lower scores in the SPPB, lower gait velocities, and shorter single-leg stance times.

An extensive neuropsychologic examination was performed in all of the patients by another author (E.S.) with 7 years of clinical experience. The neuropsychologic battery included global cognitive tests (Mini Mental State Examination; MMSE) ${ }^{27}$; executive function tests, such as Stroop ${ }^{28}$ and Trail Making Test ${ }^{29}$; and tests of the Vascular Dementia Assessment Scale, ${ }^{30}$ evaluating attentive functions such as the Maze Task, Digit, and Verbal Fluency Test. Increasing deficit corresponds with lower scores on the MMSE, Digit, and Verbal Fluency Tests and higher scores on the Stroop and Trail Making Tests and Maze Task.

The results of the motor and neuropsychologic assessment of the 36 patients are reported in Table 1 . All of the patients gave their informed consent to participate in the study.

\section{MR Acquisition Protocol}

All of the examinations were performed on a 1.5T system (Intera, Philips Medical System, Best, the Netherlands) equipped with 30$\mathrm{mT} / \mathrm{m}$ gradients and a sensitivity-encoding (SENSE) head coil.

MR Imaging. After scouts, the examination protocol included axial 3D T1-weighted turbo gradient-echo ( $\mathrm{TR}=25 \mathrm{~ms}$; TE $=4.6 \mathrm{~ms}$; flip angle $=30^{\circ} ; \mathrm{FOV}=256 \mathrm{~mm}$; matrix size $=256 \times 256 ; 160$ contiguous sections; section thickness $=1 \mathrm{~mm}$; NEX $=1$ ) images and axial T2-weighted images, which were obtained with a FLAIR sequence $(\mathrm{TR}=6000 \mathrm{~ms} ; \mathrm{TE}=120 \mathrm{~ms}$; inversion time $=2000 \mathrm{~ms}$; FOV $=250 \mathrm{~mm}$; matrix size $=256 \times 256 ; 24$ sections; section gap $=$ $0.5 \mathrm{~mm}$; section thickness $=5 \mathrm{~mm}$; turbo factor $=24$; SENSE factor $=1.75$; NEX $=2$ ).

DTI. A diffusion-weighted single-shot echo-planar imaging sequence $(\mathrm{TR}=4432 \mathrm{~ms}$; TE $=89 \mathrm{~ms}$; FOV $256 \mathrm{~mm}$; matrix size $=$ $128 \times 128$; 26 sections; section thickness $=5 \mathrm{~mm}$; no gap; $\mathrm{NEX}=2$ ) was acquired on axial plane with diffusion sensitizing gradients applied along 32 noncollinear directions using $b$ value of 0 ( $B_{0}$ image) and $1000 \mathrm{~s} / \mathrm{mm}^{2}$. Maps of MD and FA were calculated from the diffusion tensor images by Philips commercially available software.

\section{Image Analysis}

Visual Assessment. One operator (A.G.) with 5 years of experience in clinical MR imaging evaluated the FLAIR images of the 36 patients to rate LA extent by using the visual scale proposed by Fazekas et al. ${ }^{31}$ The scale has a range between 0 and 6 (On-line Table) and showed a very good interoperator agreement in a previous study. ${ }^{19}$ In addition, the operator was requested to specifically assess the signal intensity of the corpus callosum in FLAIR images.

Brain Volume Analysis. To explore possible correlation between atrophy and clinical parameters, the SIENAX method, ${ }^{32}$ part of FSL 3.3 (FMRIB, Oxford, UK), ${ }^{33}$ was applied to each subject T1-weighted acquisition to estimate the total, gray matter, and white matter brain volume, normalized for head size of the subject.

Histogram and Voxel-Based Analyses. The methods for histogram analysis were reported previously. ${ }^{24}$ Using a custom-made software (available on request) the 25th and 50th (median) percentile values, kurtosis, and skewness of the whole-brain MD and FA histograms were computed. Kurtosis describes how sharply peaked a histogram is compared with the histogram of a normal distribution. Accordingly, whereas a normal distribution has a kurtosis of 0 , a more peaked histogram has a positive kurtosis value. Skewness describes the degree of asymmetry of a histogram: a perfectly symmetric histogram has a skewness of 0 , a histogram with a long right tail has a positive skewness, whereas a negative skewness is due to the presence of a long left tail. The methods for voxel-based morphometry (VBM) and voxel-based analyses of the MD and FA maps were reported previously. ${ }^{24,34}$

\section{Statistical Methods}

The nonparametric Spearman rank test was used to assess possible correlation of the visual scores with the brain volumes and histogram parameters derived from MD and FA maps. The same test was also used to investigate possible correlation between the above MR variables and the clinical scale scores. For all of these analyses, the significance threshold was set at $P<.01$.

The correlation tool of the SPM2 software (Wellcome Department of Imaging Neuroscience, London, UK) was used to correlate maps of T1, MD, and FA (decreased gray matter, decreased white matter, increased MD, and decreased FA) with the parametric motor 

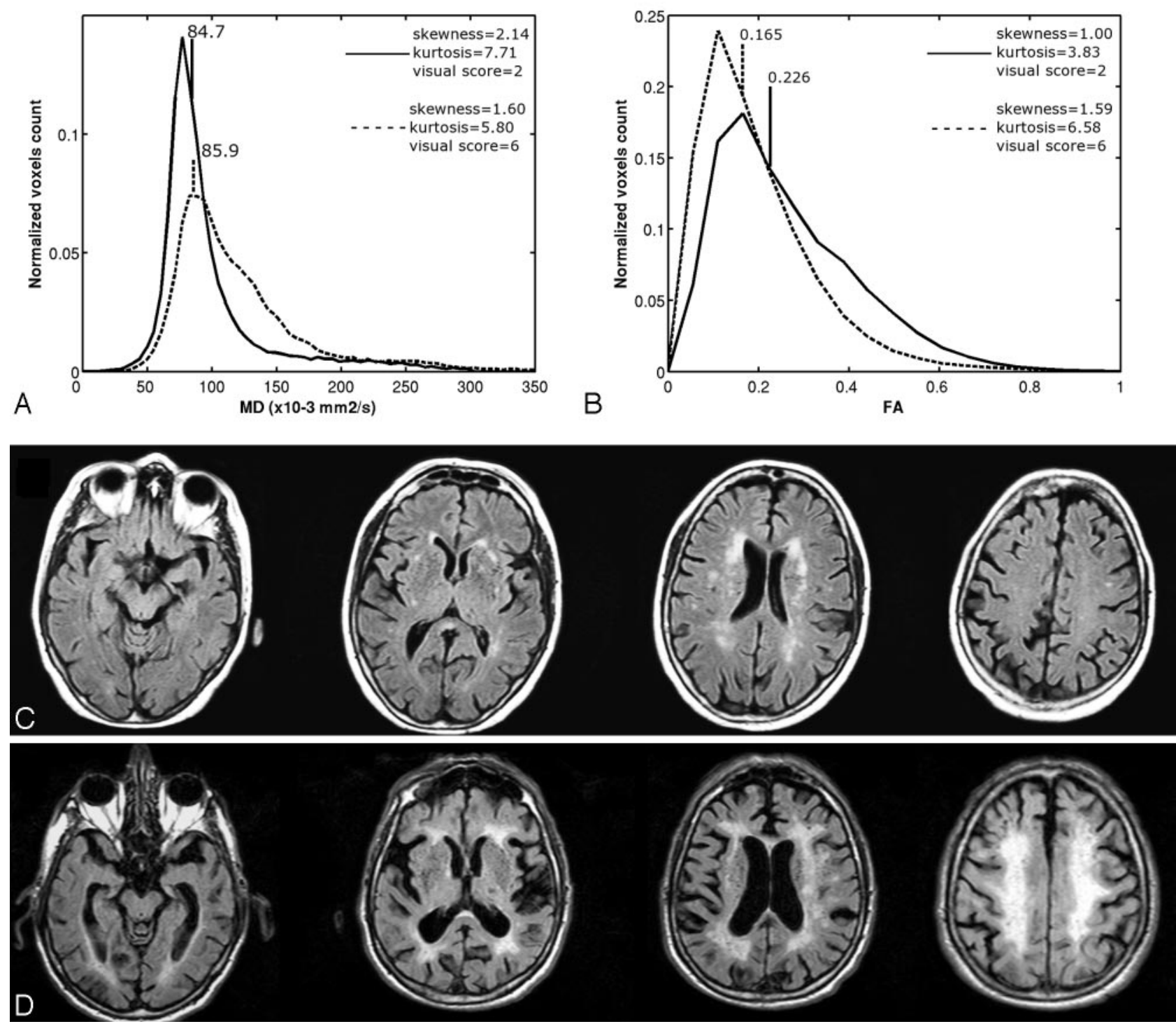

Fig 1. $A-D$, Whole-brain $\mathrm{MD}(A)$ and $F A(B)$ histograms in a patient with low $(n=2)$ visual score of $L A$ (continuous line), as shown by corresponding FLAIR images $(C)$, and in a patient with high $(n=6)$ visual score of LA (dashed line), as shown by corresponding FLAIR images $(D)$.

$A$ and $B$, The patient with the higher visual score shows higher 50 th percentile $(85.9$ vs. 84.7$)$ and lower kurtosis and skewness of the MD histogram ( $A$ ) and lower 50 th percentile $(0.165$ vs. 0.226) and higher kurtosis and skewness of the FA histogram (B) compared to the patient with lower visual score.

and neuropsychologic data. Statistical significance was set at $P<.05$ corrected for multiple comparison using the false discovery rate method. ${ }^{35}$ Because previous studies reported a correlation between atrophy of the corpus callosum and gait disorder and cognitive deficits in patients with LA, ${ }^{36-38}$ we also performed a correlation between FA maps and motor scores and between MD and FA maps and cognitive scores using a small volume correction. This was accomplished by selecting the voxels of interest corresponding with the Talairachbased mask of the corpus callosum provided by the Wake Forest University PickAtlas (Winston Salem, NC). ${ }^{39}$

\section{Results}

Figure 1 shows FLAIR images and corresponding whole-brain $\mathrm{MD}$ and FA histograms in 2 representative patients with mild (visual score 2) and extensive (visual score 6) LA. The results of visual assessment of LA; the global, gray, and white matter volumes; and the metrics of the MD and FA whole-brain histograms in the 36 patients are detailed in Table 2.
Hyperintensity of the corpus callosum in FLAIR images was observed in 2 patients only. The visual score correlated with some DTI metrics, including the 25th percentile of MD and skewness and kurtosis of FA, but not with any of the brain volumes (Table 3).

The correlation of the visual score, brain volumes, and the metrics of the MD and FA whole-brain histograms with the clinical scales scores are detailed in Table 4. No significant correlation was observed between visual score or brain volumes and any of the clinical parameters. The 25th and 50th percentiles of MD and the kurtosis and skewness of FA histograms correlated with motor and cognitive deficits. No correlation between gray or white matter volume and motor or cognitive scores were observed in the VBM analysis.

Table 5 reports the clusters of MD and FA changes significantly correlated with clinical parameters. A correlation between a wide cluster of MD change in the corpus callosum and pericallosal white matter and the SPPB score and the usual gait 
Table 2: Results of visual assessment, brain volumes, and MD and FA histogram parameters in $\mathbf{3 6}$ patients with LA

\begin{tabular}{lccc}
\hline Variable & Mean & SD & Range \\
\hline Fazekas scale & 4 & 1.3 & $2-6$ \\
Normalized brain volume, $\mathrm{mm}^{3}$ & $1,418,885$ & 114,852 & $1,135,915-1,649,413$ \\
Gray matter, $\mathrm{mm}^{3}$ & 678,719 & 177,773 & $430,615-1,325,915$ \\
White matter, $\mathrm{mm}^{3}$ & 740,166 & 242,657 & $83,385-1,106,288$ \\
Whole-brain $\mathrm{MD}, \times 10^{-3} \mathrm{~mm}^{2} / \mathrm{s}$ & & & \\
$\quad$ 25th percentile & 77.9 & 2.9 & $73.3-86.0$ \\
$\quad$ 50th percentile & 89.3 & 3.8 & $83.4-98.7$ \\
$\quad$ Skewness & 2 & 0.3 & $1-3$ \\
$\quad$ Kurtosis & 8 & 1.8 & $5-13$ \\
Whole-brain FA & & & \\
$\quad$ 25th percentile & 0.13 & 0.02 & $0.10-0.18$ \\
$\quad$ 50th percentile & 0.21 & 0.02 & $0.16-0.26$ \\
$\quad$ Skewness & 1 & 0.2 & $1-2$ \\
$\quad$ Kurtosis & 5 & 0.8 & $4-7$ \\
\hline
\end{tabular}

Note:- LA indicates leukoaraiosis; MD, mean diffusivity; FA, fractional anisotropy.

velocity was observed (Fig 2). A correlation between smaller clusters of FA in the corpus callosum and the same measurements of motor performance was observed only when small volume correction was used (Fig 3). No correlation with the scores of the cognitive tests was observed for voxel-based analysis of $\mathrm{MD}$ or FA maps without or with small volume correction.

\section{Discussion}

The clinical relevance of LA with respect to cognitive and motor functions is not established, and some discrepancy might in part arise from the different methods proposed for the assessment of LA. ${ }^{40}$

Several visual scales were proposed to quantify the extent of LA $^{40}$ with variable score spans and interobserver reproducibility. Overall, no or weak correlation with quantitative measurements of cognitive deficit was observed in cross-sectional studies evaluating a small sample of patients, ${ }^{5-7,13-15}$ whereas on greater sample sizes, typically of hundreds of patients, correlation among the extent of LA, global cognitive impairment, executive dysfunctions, and slowing of mental processing was observed. ${ }^{9,41}$ Similar results were obtained when the extent of LA was calculated using a volumetric approach. ${ }^{6,16,42}$ Few cross-sectional studies reported a correlation between the extent of LA evaluated visually or with computation of LA volume and gait or motor deficit. . $^{811,12,16}$

We failed to identify any correlation between the extent of LA and clinical scores of motor or cognitive deficit in our small cross-sectional study. This confirms that visual scales are not very sensitive to the clinical counterpart of LA. ${ }^{16}$

It is noteworthy that the visual and volumetric approaches do not assess the severity of the tissue structural changes associated with LA and discard the abnormalities in the normalappearing white matter in patients with LA. Hence, methods assessing the whole brain, including the normal-appearing white matter and the gray matter, could be more sensitive. Histogram and voxel-based analyses are 2 methods suitable for this purpose, which, despite their respective advantages and drawbacks, share a very robust statistical power. In fact, both enable analysis of many thousand of voxels compared with the few voxels usually sampled using small regions of interest.
Table 3: Spearman rank correlation $(\rho)$ values among visual score, brain volumes, and histogram parameters in $\mathbf{3 6}$ patients with LA

\begin{tabular}{lcc}
\hline Variable & Fazekas Scale, $\rho$ & $P$ \\
\hline Normalized brain volume & 0.06 & \\
Gray matter & 0.17 & \\
White matter & -0.04 & .003 \\
Whole-brain MD & & \\
25th percentile & 0.47 & \\
50th percentile & 0.36 & \\
Skewness & -0.30 & \\
$\quad$ Kurtosis & -0.23 & .003 \\
Whole-brain FA & & $<.001$ \\
$\quad$ 25th percentile & -0.15 & \\
50th percentile & -0.27 & \\
Skewness & 0.46 & \\
Kurtosis & 0.54 & \\
\hline Note:-LA indicates leukoaraiosis; MD, mean diffusivity; FA, fractional anisotropy.
\end{tabular}

In the histogram approach, an effective graphic representation of the whole-brain distribution of the parameters being investigated, such as MD or FA, is provided. It is noteworthy that histogram metrics along with evaluation of the entire brain reflect not only extent but also severity of the damage associated with LA. Whole-brain histograms were initially used to investigate correlates of disability in multiple sclerosis (MS), which is a common inflammatory white matter disease. ${ }^{43}$ The main limitation of histogram analysis is the loss of the topographic information, which, however, can be regained if the histogram calculation is preceded by segmentation based on anatomic landmarks. ${ }^{44}$

Voxel-based analysis was initially developed to assess whole-brain T1-weighted images (VBM) searching for loss of volume of the gray matter and white matter. ${ }^{34} \mathrm{VBM}$ has found application in a number of diseases to determine loss of bulk of the gray and white matter. ${ }^{21,45}$ Voxel-based analysis can also be used to assess other MR parameters, such as the ADC, MD, and $\mathrm{FA},{ }^{22-24}$ which assess the structural integrity of the remaining nervous tissue. The voxel-based approach overcomes the limitations of the region of interest approach, namely the operator dependence and the a priori knowledge bias, and maintains the regional information. Its main drawback is that the method is suited for analyzing groups of patients and not the single subject.

To the best of our knowledge, our is the first study investigating motor and cognitive correlates of LA using whole-brain histogram and voxel-based analyses of DTI. Overall, our whole-brain histogram DTI results are in line with those reported in MS. ${ }^{43}$ In particular, we observed a correlation between increasing extent of the visually appreciable LA, on the one hand, and increase of the 25th and 50th percentiles combined with decreased kurtosis and skewness of the MD and decrease of the 25th and 50th percentiles combined with increased kurtosis and skewness of the FA, on the other hand. The interpretation of these histogram metrics was addressed previously in a study of MS and can be reasonably applied to our findings. ${ }^{43}$ In particular, because normal gray and white matter show very similar MD values, the corresponding whole-brain MD histogram shows a bell-shaped curve pointing to a distribution around a central bin. The increased MD of the white matter associated with LA determines along with increase of the 25th and 50th percentiles a decrease of the 


\begin{tabular}{|c|c|c|c|c|c|c|c|c|c|}
\hline Variable & SPPB & $\begin{array}{c}\text { Usual Gait } \\
\text { Velocity, m/s }\end{array}$ & $\begin{array}{c}\text { Single-Leg Stance } \\
\text { Time, s }\end{array}$ & MMSE & $\begin{array}{l}\text { Stroop } \\
\text { Test }\end{array}$ & $\begin{array}{l}\text { Trail-Making } \\
\text { Test } \\
\end{array}$ & $\begin{array}{c}\text { Maze } \\
\text { Task }\end{array}$ & Digit & $\begin{array}{l}\text { Verbal } \\
\text { Fluency }\end{array}$ \\
\hline Fazekas scale & -0.18 & -0.15 & -0.26 & -0.19 & 0.13 & 0.08 & 0.11 & -0.23 & -0.28 \\
\hline Normalized brain volume & 0.38 & 0.02 & -0.22 & -0.05 & -0.09 & -0.08 & -0.18 & 0.15 & -0.04 \\
\hline Gray matter & -0.01 & -0.11 & -0.11 & -0.10 & -0.14 & 0.07 & 0.17 & -0.10 & 0.06 \\
\hline White matter & 0.28 & 0.26 & -0.04 & -0.02 & 0.02 & -0.14 & -0.29 & 0.24 & -0.05 \\
\hline \multicolumn{10}{|l|}{ Whole-brain MD } \\
\hline 25th percentile & -0.21 & -0.32 & $\begin{array}{c}-0.48 \\
(P=.006)\end{array}$ & -0.28 & $\begin{array}{c}0.51 \\
(P=.001)\end{array}$ & 0.30 & 0.32 & -0.30 & $\begin{array}{c}-0.46 \\
(P=.004)\end{array}$ \\
\hline 50th percentile & -0.15 & -0.20 & -0.35 & -0.16 & $\begin{array}{c}0.54 \\
(P<.001)\end{array}$ & 0.31 & 0.30 & -0.31 & $\begin{array}{c}-0.44 \\
(P=.007)\end{array}$ \\
\hline Skewness & -0.28 & 0.34 & 0.16 & 0.34 & -0.23 & -0.42 & $\begin{array}{c}-0.44 \\
(P=.008)\end{array}$ & $\begin{array}{c}0.45 \\
(P=.006)\end{array}$ & 0.13 \\
\hline Kurtosis & 0.24 & 0.25 & 0.16 & 0.35 & -0.17 & -0.37 & -0.38 & 0.40 & 0.06 \\
\hline \multicolumn{10}{|l|}{ Whole-brain FA } \\
\hline 25th percentile & -0.02 & -0.00 & -0.01 & -0.24 & -0.11 & -0.01 & -0.17 & -0.08 & 0.10 \\
\hline 50th percentile & 0.04 & -0.10 & 0.09 & -0.18 & -0.17 & -0.06 & -0.09 & 0.01 & 0.17 \\
\hline Skewness & -0.18 & -0.35 & -0.21 & -0.15 & 0.26 & 0.14 & 0.18 & -0.28 & -0.41 \\
\hline Kurtosis & -0.22 & -0.39 & -0.27 & -0.21 & 0.32 & 0.18 & 0.21 & -0.32 & $\begin{array}{c}-0.46 \\
(P=.005)\end{array}$ \\
\hline
\end{tabular}

Note:-LA indicates leukoaraiosis; MD, mean diffusivity; FA, fractional anisotropy; SPPB, Short Physical Performance Battery; MSSE, Mini Mental State Examination.

Table 5: Correlations between voxel-based analysis of MD and FA maps and motor assessment

\begin{tabular}{|c|c|c|c|c|c|c|c|c|}
\hline \multirow[b]{2}{*}{ Correlation } & \multirow{2}{*}{$\begin{array}{c}\text { Cluster Extent, } \\
\mathrm{mm}^{3}\end{array}$} & \multirow[b]{2}{*}{$P^{*}$} & \multirow{2}{*}{$\begin{array}{c}t \\
\text { Score }\end{array}$} & \multirow{2}{*}{$\begin{array}{c}Z \\
\text { Score }\end{array}$} & \multicolumn{3}{|c|}{$\begin{array}{l}\text { Coordinates (Local } \\
\text { Maxima) }\end{array}$} & \multirow[b]{2}{*}{ Areas } \\
\hline & & & & & $\mathrm{x}$ & y & z & \\
\hline MD, SPPB & 45648 & 0.048 & 5.58 & 4.67 & 16 & -24 & 34 & Corpus callosum and pericallosal white matter \\
\hline MD, usual gait velocity & 16536 & 0.043 & 5.14 & 4.37 & 6 & 14 & 20 & Corpus callosum and pericallosal white matter \\
\hline FA, SPPB & 824 & 0.019 & 4.09 & 3.65 & -6 & -28 & 20 & Corpus callosum splenium \\
\hline FA, usual gait velocity & 572 & 0.025 & 3.72 & 3.39 & 6 & 0 & 24 & Corpus callosum genu \\
\hline
\end{tabular}

Note:-MD indicates mean diffusivity; FA, fractional anisotropy; SPPB, Short Physical Performance Battery.

${ }^{*} P$ is false discovery rate corrected for multiple comparisons.
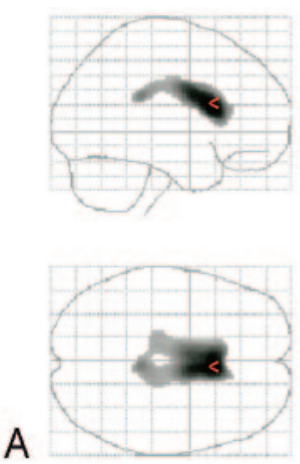

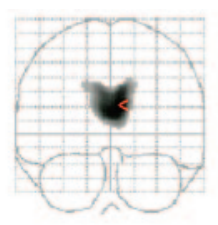

$\operatorname{SPM}\left\{T_{34}\right\}$

Fig 2. $A-C$, SPM2 "glass brain" representation showing large clusters in the corpus callosum and pericallosal white matter of significant $(P<0.05$, corrected for multiple comparison by false discovery rate method) correlation between increasing MD value and scores of motor deficit (usual gait velocity in $A$; SPPB in $B$ ) in patients with LA. Superimposition of the same cluster demonstrated in $B$ onto $\mathrm{T} 1$ template $(C)$.

kurtosis (the curve becomes less peaked) and of the skewness (the curve becomes less asymmetric) of the histogram. On the contrary, the distribution of normal gray and white matter anisotropy is very different, with lower values for the gray matter and higher values for the white matter. Hence, FA histograms created from images containing gray and white matter are necessarily the results of the superimposition of 2 different bell-shaped curves. The decreased FA of the white matter associated with LA determines along with a decrease of the 25th and 50th percentiles a relative increase of the low FA values with increased kurtosis (the curve becomes more peaked) and of the skewness (the curve becomes more asymmetric) of the histogram.

More interestingly, we found correlation between histogram metrics and quantitative clinical scores describing the motor and cognitive impairment observed in patients with LA. In particular, whereas our data confirm those of a previous region of interest study, which showed correlation between $\mathrm{MD}$ in the normal-appearing white matter and executive dysfunction in patients with LA, ${ }^{13}$ they reveal a correlation with motor dysfunction not reported previously. The fragmentary correlation between the MD and FA histogram metrics and 

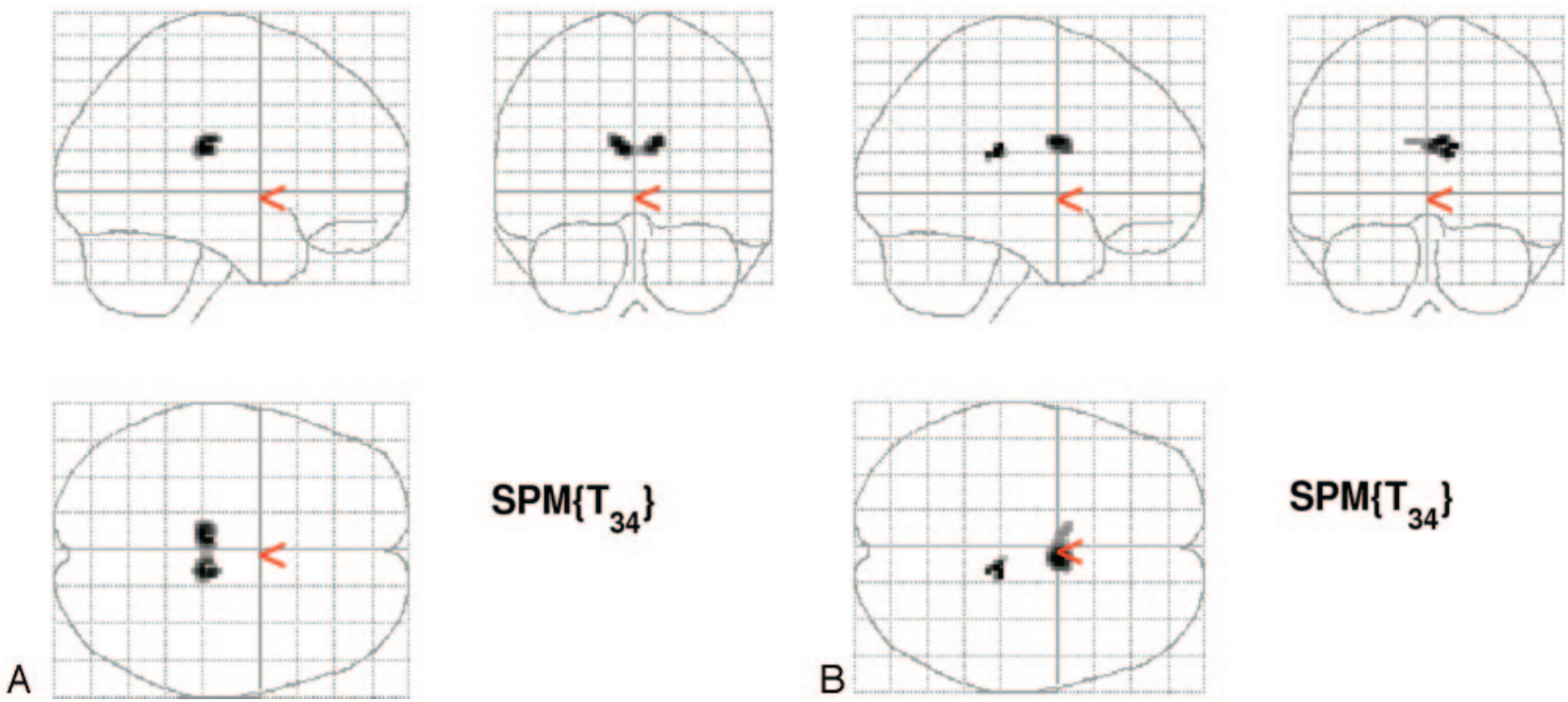

\section{$\operatorname{SPM}\left\{\mathrm{T}_{34}\right\}$}

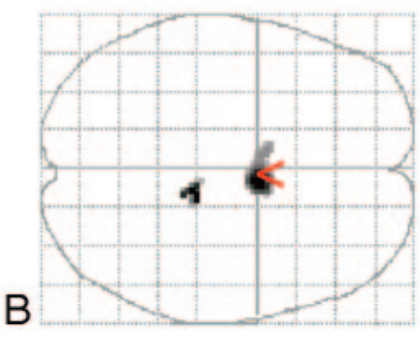

\section{$\operatorname{SPM}\left\{\mathrm{T}_{34}\right\}$}

Fig 3. $A$ and $B$, SPM2 "glass brain" representation showing small clusters in the corpus callosum of significant $(P<0.05$, corrected for multiple comparison by false discovery rate method) correlation using small volume correction between decreasing FA value and scores of motor deficit (usual gait velocity in $A$; SPPB in $B$ ) in patients with LA.

the motor and cognitive scores in our study could suggest by-chance correlation. However, we used a conservative statistical test for correlation, all of the correlations were expected, and they are in line with previous observations in studies using visual or volumetric assessment of LA.

The main result of the voxel-based analysis that we performed is the strong correlation between a wide cluster-increased $\mathrm{MD}$ in the corpus callosum and pericallosal white matter fibers and 2 indices of motor impairment. Although a correlation between corpus callosum atrophy and motor disability was reported previously in patients with LA, ${ }^{36,37}$ we did not find correlation between motor score and any cluster in the voxel-based analysis of T1-weighted images that we performed. On the other hand, in line with general sparing of the signal intensity of the corpus callosum in patients with LA, ${ }^{46}$ callosal signal intensity abnormality was observed in only 2 of our 36 patients. This indicates that we probably demonstrated a microscopic and presumably early damage of the callosal white matter fibers. We submit that this finding could explain the pathophysiology of motor disturbances in LA and, in particular, the similarity of the clinical motor disturbance of patients with LA with that of patients with so called "normal pressure hydrocephalus," in which impingement of the callosal fibers on the falx cerebri caused by dilated ventricules is assumed to be responsible for the gait "apraxia," which is reversed by CSF shunt and decreased ventricular size. ${ }^{47} \mathrm{Al}-$ though a correlation between the regional atrophy of the corpus callosum and mental slowing and executive deficits was reported recently in the entire group of the patients enrolled in the LADIS protocol, ${ }^{38}$ we failed to identify any correlation in the voxel-based analysis of the maps of the gray and white matter volume, the MD, and the FA with the neuropsychologic tests scores. We speculate that this negative result fundamentally reflects the small sample size in our study.

We recognize some limitations of our study. First, we examined a relatively small number of subjects in our study. Hence, the results that we obtained have to be considered as preliminary to future studies on larger samples of patients.
Second, we evaluated possible signal intensity changes in the corpus callosum on axial FLAIR images only. This could determine an underestimation of such signal intensity changes, which are better demonstrated on sagittal or coronal planes. Third, we did not examine healthy age-matched control subjects. However, many studies using DWI and DTI have demonstrated the progressive mild modifications of the diffusion properties of the water protons in the aging brain with increased ADC and MD and decreased FA, which are far less pronounced than those encountered in patients with LA. ${ }^{48-50}$ Fourth, the methodology that we used is not widely available. However, DTI is becoming a fundamental component of MR imaging equipment, and $\mathrm{MD}$ and FA histogram metrics showed excellent imaging-reimaging and interimaging unit reproducibility. ${ }^{51}$ The software for image processing and SPM2 are available from the Internet, and the software for computation of histogram metrics is available on request.

In conclusion, our study confirms the hypothesis that the results of whole-brain histogram and voxel-based analyses of the damage associated with LA demonstrated by DTI are more closely related to the actual clinical deficit than the visual assessment. For this reason, application of these analysis methods to the evaluation of LA should be encouraged, especially in the context of future pharamacologic trials for LA. In particular, voxel-based analysis shows that the motor impairment in patients with LA is strongly correlated with increased MD in the corpus callosum and pericallosal white matter fibers. Voxel-based analysis of MD and FA maps on larger samples has the potential to clarify many physiopathologic aspects of LA.

\section{References}

1. Basile AM, Pantoni L, Pracucci G, et al. Age, hypertension, and lacunar stroke are the major determinants of the severity of age-related white matter changes. The LADIS (Leukoaraiosis and Disability in the Elderly) Study. Cerebrovasc Dis 2006;21:315-22

2. Brown WR, Moody DM, Thore CR, et al. Apoptosis in leukoaraiosis. AJNR Am J Neuroradiol 2000;21:79-82

3. Moody DM, Thore CR, Anstrom JA, et al. Quantification of afferent vessels 
shows reduced brain vascular density in subjects with leukoaraiosis. Radiology 2004;233:883-90

4. Fazekas F, Kleinert R, Offenbacher H, et al. Pathologic correlates of incidental MRI white matter signal hyperintensities. Neurology 1993;43:1683-89

5. Schmidt R, Fazekas F, Offenbacher H, et al. Neuropsychological correlates of MRI white matter hyperintensities: a study of 150 normal volunteers. Neurology 1993;43:2490-94

6. Ylikoski R, Ylikoski A, Erkinjuntti T, et al. White matter changes in healthy elderly persons correlate with attention and speed of mental processing. Arch Neurol 1993;50:818-24

7. Almkvist $O$. White matter changes: possible impact on cognitive functions. In: Pantoni L, Inzitari D, Wallin A, eds. The Matter of White Matter. Utrecht, the Netherlands: Academic Pharmaceutical Productions; 2000;81-95

8. Guttmann CRG, Benson R, Warfield SK, et al. White matter abnormalities in mobility-impaired older persons. Neurology 2000;54:1277-83

9. De Groot JC, de Leeuw FE, Oudkerk M, et al. Cerebral white matter lesions and cognitive function: the Rotterdam Scan Study. Ann Neurol 2000;47:145-51

10. Koga H, Yuzuriha T, Yao $\mathrm{H}$, et al. Quantitative MRI findings and cognitive impairment among community dwelling elderly subjects. J Neurol Neurosurg Psychiatry 2002;72:737-41

11. Pugh KG, Lipsitz LA. The microvascular frontal subcortical syndromes of aging. Neurobiol Aging 2002;23:421-31

12. Onen F, Feugeas MC, Baron G, et al. Leukoaraiosis and mobility decline: a high resolution magnetic resonance imaging study in older people with mild cognitive impairment. Neurosci Lett 2004;355:185-88

13. O'Sullivan M, Morris RG, Huckstep B, et al. Diffusion tensor MRI correlates with executive dysfunction in patients with ischaemic leukoaraiosis. J Neurol Neurosurg Psychiatry 2004;75:441-47

14. Ross ED, Hansel SL, Orbelo DM, et al. Relationship of leukoaraiosis to cognitive decline and cognitive aging. Cog Behav Neurol 2005;18:89-97

15. Van der Flier WM, van Straaten ECW, Barkhof F, et al. Medial temporal lobe atrophy and white matter hyperintensities are associated with mild cognitive deficits in non-disabled elderly people: the LADIS study. J Neurol Neurosurg Psychiatry 2005;76:1497-500

16. Van Straaten ECW, Fazekas F, Rostrup E, et al. Impact of white matter hyperintensities scoring method on correlations with clinical data. The LADIS study. Stroke 2006;37:836-40

17. Chabriat H, Pappata S, Poupon C, et al. Clinical severity in CADASIL related to ultrastructural damage in white matter. In vivo study with diffusion tensor MRI. Stroke 1999;30:2637-43

18. O'Sullivan M, Summers PE, Jones DK, et al. Normal-appearing white matter in ischemic leukoaraiosis: a diffusion tensor MRI study. Neurology 2001;57: 2307-10

19. Mascalchi M, Tessa C, Moretti M, et al. Whole brain apparent diffusion coefficient histogram: a new tool for evaluation of leukoaraiosis. J Magn Reson Imaging 2002;15:144-48

20. Mascalchi M, Moretti M, Della Nave R, et al. Longitudinal evaluation of leukoaraiosis with whole brain ADC histograms. Neurology 2002;59:938-40

21. Thieben MJ, Duggins AJ, Good CD, et al. The distribution of structural neuropathology in pre-clinical Huntington's disease. Brain 2002;125:1815-28

22. Leung LH, Ooi GC, Kwong DL, et al. White-matter diffusion anisotropy after chemo-irradiation: a statistical parametric mapping study and histogram analysis. NeuroImage 2003;21:261-68

23. Xie S, Xiao JX, Gong GL, et al. Voxel-based detection of white matter abnormalities in mild Alzheimer disease. Neurology 2006;66:1845-49

24. Della Nave R, Magaudda A, Michelucci R, et al. Whole brain histogram and voxel-based analyses of ADC and MTR in the coeliac disease, epilepsy and cerebral calcifications syndrome. AJNR Am J Neuroradiol 2007;28:479-85

25. Pantoni L, Basile AM, Pracucci G, et al. Impact of age-related cerebral white matter changes on the transition to disability-the LADIS study: rationale, design and methodology. Neuroepidemiology 2005;24:51-62

26. Guralnik JM, Simonsick EM, Ferrucci L, et al. A short physical performance battery assessing lower extremity function: association with self-reported disability and prediction of mortality and nursing home admission. J Gerontol 1994;49:M85-94

27. Folstein MF, Folstein SE, McHugh PR. "Mini-mental state.” A practical method for grading the cognitive state of patients for the clinician. J Psychiatr Res 1975;12:189-98

28. McLeod CM. Half a century of research on the Stroop effect: an integrative review. Psychol Bull 1991;109:163-203

29. Reitan RM. Validity of the Trail Making Test as an indicator of organic brain damage. Percep Mot Skills 1958;8:271-76

30. Ferris SH. General measures of cognition. Int Psychogeriatr 2003;15(supp 1):215-17

31. Fazekas F, Chawluk JB, Alavi A, et al. MR signal abnormalities at $1.5 \mathrm{~T}$ in Alzheimer's dementia and normal aging. AJNR Am J Neuroradio 1987;149:351-56

32. Smith SM, Zhang Y, Jenkinson M, et al. Accurate, robust and automated longitudinal and cross-sectional brain change analysis. NeuroImage 2002;17: $479-89$

33. Smith SM, Jenkinson M, Woolrich MW, et al. Advances in functional and structural MR image analysis and implementation as FSL. NeuroImage 2004:23:208-19

34. Good CD, Johnsrude IS, Ashburner J, et al. A voxel-based morphometric study of ageing in 465 normal adult human brains. NeuroImage 2001;14:21-36

35. Genovese CR, Lazar NA, Nichols T. Thresholding of statistical maps in functional neuroimaging using the false discovery rate. NeuroImage 2002;15 $870-78$

36. Moretti M, Carlucci G, Di Carlo A, et al. Corpus callosum atrophy is associated with gait disorders in patients with leukoaraiois. Neurol Sci 2005;26:61-66

37. Ryberg C, Rostrup E, Stegmann MB, et al. Clinical significance of corpus callosum atrophy in a mixed elderly population. Neurobiol Aging In press

38. Jokinen $\mathrm{H}$, Ryberg $\mathrm{C}$, Kalska $\mathrm{H}$, et al. Corpus callosum atrophy is associated with mental slowing and executive deficits in subjects with age-related white matter hyperintensities. The LADIS study. J Neurol Neurosurg Psychiatry In press

39. Maldjian JA, Laurienti PJ, Kraft RA, et al. An automated method for neuroanatomic and cytoarchitectonic atlas-based interrogation of fMRI data sets. $\mathrm{Neu}$ roImage 2003;19:1233-39

40. Pantoni L, Simoni M, Pracucci G, et al. Visual rating scales for age-related white matter changes (leukoaraiosis). Can the heterogeneity be reduced? Stroke 2002;33:2827-33

41. Pantoni L, Poggesi A, Basile AM, et al. Leukoaraiosis predicts hidden global functioning impairment in nondisabled older people: the LADIS (leukoaraiosis and disability in the elderly) study. J Am Geriatr Soc 2006;54:1095-101

42. Jack CR, O'Brien PC, Rettman DW, et al. FLAIR histogram segmentation for measurement of leukoaraiosis volume. J Magn Reson Imaging 2001;14:668-76

43. Cercignani M, Inglese M, Pagani E, et al. Mean diffusivity and fractional an isotropy histograms of patients with multiple sclerosis. AJNR Am J Neuroradiol 2001;22:952-58

44. Bozzali M, Franceschi M, Falini A, et al. Quantification of tissue damage in AD using diffusion tensor and magnetization transfer MRI. Neurology 2001;57: 1135-37

45. Karas GB, Burton EJ, Rombouts SA, et al. A comprehensive study of gray matter loss in patients with Alzheimer's disease using optimized voxel-based morphometry. NeuroImage 2003;18:895-907

46. Moody MD, Bell MA, Challa VR. The corpus callosum, a unique white matter tract: anatomic features that may explain sparing in Binswanger disease and resistance to flow of fluid masses. AJNR Am J Neuroradiol 1988;9:1051-59

47. Jinkins JR. Clinical manifestations of hydrocephalus caused by impingement of the corpus callosum on the falx: an MR study in 40 patients. AJNR Am J Neuroradiol 1991;12:331-40

48. Chun T, Filippi CG, Zimmerman RD, et al. Diffusion changes in the aging human brain. AJNR Am J Neuroradiol 2000;21:1078-83

49. Nusbaum AO, Tang CY, Buchsbaum MS, et al. Regional and global changes in cerebral diffusion with normal aging. AJNR Am J Neuroradiol 2001;22:136-42

50. Rovaris M, Iannucci G, Cercignani M, et al. Age-related changes in conventional, magnetization transfer, and diffusion-tensor MR imaging findings: study with whole-brain tissue histogram analysis. Radiology 2003;227:731-38

51. Cercignani M, Bammer R, Sormani MP, et al. Inter-sequence and inter-imaging unit variability of diffusion tensor MR imaging histogram-derived metrics of the brain in healthy volunteers. AJNR Am J Neuroradiol 2003;24:638-43 